\title{
IMPLEMENTACIÓN DE UNA RED DE ALERTA DE EMERGENCIA POR LA OCURRENCIA DE AMENAZAS NATURALES Y ANTRÓPICAS USANDO SUBPORTADORAS DE TELEVISIÓN DIGITAL
}

\author{
IMPLEMENTATION OF AN EMERGENCY ALERT \\ NETWORK BY OCCURRENCE OF A NATURAL AND \\ HUMAN THREATS USING DIGITAL TELEVISION \\ SUBCARRIERS
}

\author{
Ángel Gustavo Orna Proaño(1), Edwin Marcelo Cevallos Romero ${ }^{(2)}$ y Charles \\ Edisson Escobar Terán ${ }^{(3)}$ \\ (1) (3) Pontificia Universidad Católica del Ecuador \\ agopdrt@hotmail.com \\ cescobar637@puce.edu.ec \\ (2) Universidad de las Fuerzas Armadas \\ emcevallos@espe.edu.ec
}

RESUMEN

Las redes de alerta de emergencia nacen como necesidad de los organismos encargados de precautelar por la seguridad ciudadana, para tomar oportunamente las medidas correspondientes al detectar la ocurrencia de amenazas naturales y antrópicas, que pudieran afectar a sus territorios y sobre todo a los seres humanos que los habitan; esto ha hecho que se desarrollen sistemas aprovechando los avances tecnológicos en las redes de telecomunicaciones, permitiendo detectar eventos casi al instante de producirse y enviar información a esos organismos; a pesar de toda la tecnología utilizada en la detección oportuna de estos eventos, la última etapa, y quizá la más importante, que es la difusión de esas alertas a la ciudadanía que pudiera ser afectada, todavía no es todo lo eficiente y eficaz que debería ser.

La comparación entre las distintas alternativas tecnológicas utilizadas para la implementación de sistemas de alerta de emergencia en diferentes partes del mundo, incluyendo los que se está instalando en cantones como Quito, Rumiñahui y Latacunga, que ason sistemas compuesto de sirenas de largo alcance, ubicadas en sectores estratégicos, permite concluir que no cubriría a toda la población de los territorios amenazados, reduciendo las posibilidades a los habitantes de tomar medidas para precautelar sus bienes materiales y sus vidas. Es así que para reducir ese inconveniente aparece la televisión digital terrestre como una solución complementaria a otros sistemas de alerta, con la diferencia que la emisión se la realizaría a una gran área de cobertura, en forma simultánea y de manera sectorizada, evitando alertar innecesariamente a poblaciones que podrían 
desviar la atención de los organismos de seguridad y socorro de las zonas verdaderamente necesitadas.

Palabras Claves: Sistema de Alerta de Emergencia, Amenazas Naturales y Antrópicas, Televisión Digital, Subportadoras

\section{ABSTRACT}

Networks emergency alert born as a need for agencies protectively for public safety, to take timely appropriate action to detect the occurrence of natural and anthropic hazards that could affect their territories and especially humans who inhabit; this has meant that systems are developed taking advantage of technological advances in telecommunications networks, allowing almost instantly detect events occur and send information to these agencies; despite all the technology used in the early detection of these events, the last stage, and perhaps most important, which is the spread of those alerts to the public that could be affected is not yet as efficient and effective should be.

The comparison between the different technological alternatives used for the implementation of early warning systems emergency in different parts of the world, including being are installed in cantons such as Quito, Rumiñahui and Latacunga, which is a system composed of sirens of long range, located in strategic sectors to the conclusion that not cover the entire population of the threatened territories, reducing the chances the inhabitants to take measures to forewarn their property and their lives. Thus digital terrestrial television to reduce this drawback appears as a complementary solution to other warning systems, with the difference that the emission would accomplish a large coverage area, simultaneously and sectored, avoiding alerting unnecessarily populations that could divert attention from security agencies and relief of the truly needy areas.

Key words: Emergency Warning System, Natural and Man-made Hazards, Digital Television, Subcarrier.

\section{INTRODUCCIÓN}

En los últimos años, el Distrito Metropolitano de Quito (DMQ), se ha visto afectado por la ocurrencia de eventos naturales como las erupciones de los volcanes Pichincha y Cotopaxi, inundaciones por la llegada del Fenómeno del Niño, así como eventos antrópicos aislados generados por el propio ser humano como incendios forestales, roturas del oleoducto y otros que han obligado que de parte de los distintos Organismos locales y nacionales encargados de la seguridad de la ciudadanía como el Ministerio de Seguridad, la Secretaría de Gestión de Riesgos, el Municipio del DMQ y el ECU 911, con la ayuda de las Fuerzas Armadas, la Policía Nacional y el Cuerpo de Bomberos, hayan implementado sistemas de emergencia que puedan alertar oportunamente a la población que podría verse afectada tanto en bienes materiales como en pérdida de vidas humanas.

Recientemente en el año 2014 se presentó en el DMQ la amenaza de la erupción del volcán Cotopaxi, ante lo cual se implementó el "sistema de alarmas 
tempranas" (SAT) instalado por parte del Municipios del DMQ, el cual consta de sirenas cuyo sonido oscila entre $115 \mathrm{~dB}$ y $121 \mathrm{~dB}$ que pueden ser escuchados a $2.5 \mathrm{Km}$ a la redonda, calculándose de parte de las instituciones encargadas de este tema que el tiempo promedio para que las personas vayan a sitios seguros 0 albergues, después de escuchar las sirenas será de entre 15 y 30 minutos. La figura 1a muestra una de estas sirenas. A pesar de que con el SAT (6 a marzo 2016), que cubre una gran cantidad de sectores en los valles cercanos a la ciudad de Quito se podría alertar a los pobladores de los mismos, surgen una serie de interrogantes, enfocadas a la cobertura efectiva de estos sistemas y qué porcentaje de la población se beneficia o no.

Con el análisis realizado, se considera a la televisión digital como una alternativa para la implementación de una red de alerta de emergencia, por el manejo de los datos a ser transmitidos utilizando las subportadoras que se emiten al aire y aprovechando esa característica de "difusión" (broadcast), que tienen las señales de radiodifusión que permite cubrir grandes extensiones con un retraso imperceptible en relación a la señal de origen, con una emisión de alta calidad, libre de interferencias y de afectaciones por ruidos radioeléctricos e impulsivos, y además por la posibilidad de zonificación de las áreas de afectación gracias al empleo de un sistema de modulación y multiplexación robusto y eficiente.

El país cuenta con 23 señales de TDT al aire distribuidas en Quito y Guayaquil donde se encuentran las principales cadenas de televisión que operan con señales analógicas; estas cadenas son las que actualmente están levantando una señal de televisión digital en "simulcast" (transmisión simultánea) con su señal analógica, por tanto se puede contar con una infraestructura básica.

\section{VULNERABILIDAD DEL DMQ}

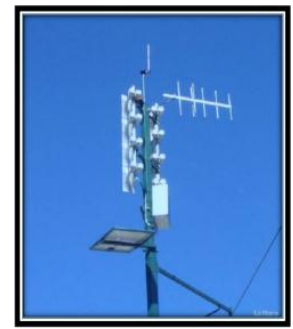

a)

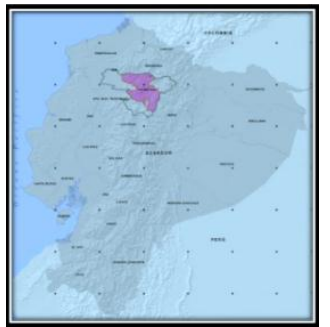

b)

Figura 1 a) Sirenas de la red SAT del DMQ. Fuente: Diario La Hora. b) Ubicación geográfica del DMQ. Fuente: Atlas de Amenazas Naturales del DMQ 2015

La ciudad de Quito, está situada al norte de Ecuador, como indica la figura $1 \mathrm{~b}$, es parte de la provincia de Pichincha y ocupa una superficie de $4.235,2 \mathrm{Km}^{2}$, acoge al $15.5 \%$ de la población del Ecuador (2.505.344 habitantes al 2105 según el INEC censo 2010), y por los servicios que ofrece ha crecido en forma desordenada, especialmente por asentamientos humanos que se ubican en zonas de elevada vulnerabilidad como laderas y cuencas hidrográficas (quebradas y ríos). Según los datos de la Secretaría General de Seguridad y Gobernabilidad del DMQ, las inundaciones, los incendios forestales y los movimientos en masa son los fenómenos más recurrentes. Adicionalmente, se considera también las erupciones volcánicas y los sismos que son otras amenazas de origen natural y que podrían presentarse. 


\section{SISTEMAS DE ALERTA DE EMERGENCIA}

A lo largo de la historia las amenazas naturales y antrópicas han costado vidas e infraestructura, la cuantificación del daño causado por los mismos no está relacionada solo a su gravedad, sino también a la capacidad de reacción de las personas que viven en zonas vulnerables, por esta razón los esfuerzos para reducir el riesgo de desastres se ha centrado, en el desarrollo de sistemas de alerta de emergencia.

Los sistemas de alerta se componen fundamentalmente de cuatro elementos; 1) Vigilancia técnica permanente, 2) Conocimiento de los posibles riesgos, 3) Difusión de alertas a la ciudadanía, 4) Preparación de la población para reaccionar.

Lo bien que funcionen esos sistemas dependerá de la base científica sólida para la predicción y el pronóstico de los eventos que se presenten y la capacidad de realizar monitoreos las 24 horas del día en todo el año.

\subsection{Tecnologías de vigilancia y alerta}

La continua aparición de eventos naturales y los avances tecnológicos han hecho que en el mundo se desarrollen una serie de tecnologías para la prevención y detección de los eventos naturales, con el objeto de alertar oportunamente a la ciudadanía. A continuación se citan varias de esas tecnologías.

- Previsión y modelización.

- Teledetección y sistemas de información geográfica

- Comunicación por satélite.

- Telefonía móvil.

- TIC para el crowdsourcing 1.

- Ushahidi

\section{LA TELEVISIÓN DIGITAL}

La televisión digital terrestre es la tecnología utilizada para la transmisión de señales de televisión que ha generado mucha expectativa en los países donde se la ha implementado, por cuanto a más de ofrecer una mejor calidad de la señal de televisión, introduce servicios adicionales como interactividad, movilidad y aplicaciones en línea. Figura 2a.

Los receptores de televisión analógica no son capaces de interpretar las señales del sistema digital. Es necesaria la conversión, realizada por equipos conocidos como Set-Top-Box-STB. Actualmente existen en el mercado televisores que tienen embebido en su sistema un bloque de decodificación que les permite recibir señales analógicas y digitales por lo tanto ya no se requiere de un STB independiente. En la figura $2 b$ se muestra el despliegue de la televisión digital en el mundo.

1 Crowdsourcing, definido como una "colaboración abierta" que permite la externalización de tareas a un grupo numeroso de personas.

${ }^{2}$ En lengua Swahili (hablada en Kenia y Tanzania) significa "testigo o testimonio" 


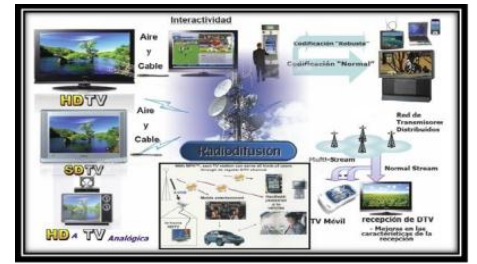

a)

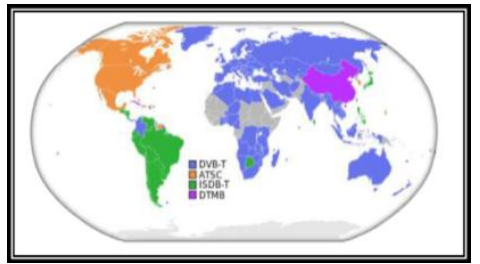

b)

Figura 2 a) Escenarios de la TDT. Fuente: SUPERTEL ${ }^{3}$; b) Despliegue de la TDT. Fuente:https://es.wikipedia.org/wiki/Televisi\%C3\%B3n_digital_terrestre

\subsection{TDT en Ecuador}

Luego del proceso de pruebas técnicas realizadas en la ciudad de Quito entre los años 2008 y 2009, y la emisión de informes socioeconómicos y de cooperación internacional, respecto a los diferentes estándares de TDT, por parte de la ex Superintendencia de Telecomunicaciones (SUPERTEL), el ex Consejo Nacional de Telecomunicaciones (CONATEL) mediante Resolución 084-05CONATEL-2010 del 25 de marzo del 2010, resolvió acoger los informes de la ex SUPERTEL y adoptar el estándar de TDT brasileño japonés-ISDB-Tb, para implementarse en el Ecuador.

\subsubsection{Espectro radioeléctrico y canalización}

\subsubsection{Bandas de frecuencias}

Conforme lo establecido en la "Norma Técnica para el Servicio de Radiodifusión de Televisión Abierta Analógica", emitida mediante Resolución ARCOTEL-2015-0218 del 4 de agosto del 2015, la banda de frecuencias que se usará para la transmisión de TDT en el Ecuador es la banda UHF del espectro radioeléctrico, atribuida para el Servicio de Radiodifusión con emisiones de Televisión. Figura 3a.

Tabla 1. Bandas de frecuencias para televisión UHF.

\begin{tabular}{|c|c|c|}
\hline \multirow{2}{*}{ Banda IV } & $470-476 \mathrm{MHz}$ & Canales del 14 al 15 \\
& $500-608 \mathrm{MHz}$ & Canales del 19 al 36 \\
& $614-644 \mathrm{MHz}$ & Canales del 38 al 42 \\
\hline Banda V & $644-698 \mathrm{MHz}$ & Canales del 43 al 51 \\
\hline
\end{tabular}

Durante el período de simulcast se utilizarán los canales adyacentes a los utilizados en cada zona geográfica, en la banda de canales del 19 al 51.

\subsection{2. Áreas de operación}

La Norma Técnica que para efectos de implementación de TDT emita el Organismo de Regulación, incluirá la zonificación del país para las concesiones de televisión digital, hasta tanto se considerará la zonificación de la actual "Norma Técnica para el Servicio de Radiodifusión de Televisión Abierta Analógica", emitida

\footnotetext{
${ }^{3}$ Informe sobre la Implementación de la TDT en el Ecuador - 2011
} 
mediante Resolución ARCOTEL-2015-0218 del 4 de agosto del 2015. En la que para la provincia de Pichincha, donde se encuentra el DMQ, se establece:

Tabla 2 Asignación de canales para la provincia de Pichincha. ${ }^{4}$

\begin{tabular}{|c|l|c|l|}
\hline $\begin{array}{c}\text { ÁREA DE OPERACIÓN } \\
\text { INDEPENDIENTE }\end{array}$ & $\begin{array}{c}\text { DESCRIPCIÓN DEL ÁREA DE } \\
\text { OPERACIÓN INDEPENDIENTE }\end{array}$ & $\begin{array}{c}\text { GRUPO DE } \\
\text { CANALES UHF }\end{array}$ & $\begin{array}{l}\text { ÁREAS DE OPERACIÓN } \\
\text { ZONAL }\end{array}$ \\
\hline \multirow{5}{*}{ P1 } & $\begin{array}{l}\text { Provincia de Pichincha, excepto los } \\
\text { cantones San Miguel de Los Bancos, } \\
\text { Pedro Vicente Maldonado, Puerto } \\
\text { Quito, parroquia Manuel Cornejo } \\
\text { Astorga, incluye la parroquia Mindo del } \\
\text { cantón San Miguel de Los Bancos. }\end{array}$ & G1 Y G4 & $\begin{array}{l}\text { 1. Quito, Machachi } \\
\text { (Mejía), Sangolquí } \\
\text { (Rumiñahui), Tabacundo } \\
\text { (Pedro Moncayo), } \\
\text { Cayambe } \\
\text { 2. Parroquia Mindo } \\
\text { 3. Parroquia Pacto }\end{array}$ \\
\hline
\end{tabular}

\subsubsection{Autorizaciones de carácter temporal}

Para el inicio de las transmisiones de TDT, se ha considerado la operación de estaciones de televisión a través de autorizaciones de carácter temporal, conforme lo establecido en la regulación vigente y una vez que se ha reconocido a TDT como un evento de trascendencia nacional, estas autorizaciones tienen el plazo de un año, con la posibilidad de renovación por el mismo período.

Tabla 3 Canales de TDT en Quito que cuentan con autorización temporal.

\begin{tabular}{|c|l|c|c|c|}
\hline No. & \multicolumn{1}{|c|}{ NOMBRE ESTACIÓN } & CANAL TDT & M/R & CANAL VIRTUAL \\
\hline 1 & ECUADOR TV & 47 & M & 7 \\
\hline 2 & TELEVISIÓN DEL PACIFICO & 30 & $\mathrm{M}$ & 2 \\
\hline 3 & TELEAMAZONAS & 32 & $\mathrm{M}$ & 4 \\
\hline 4 & TELESISTEMA & 34 & $\mathrm{M}$ & 5 \\
\hline 5 & TELEVISORA NACIONAL & 36 & $\mathrm{M}$ & 8 \\
\hline 6 & TELEVISIÓN SATELITAL & 39 & $\mathrm{M}$ & 25 \\
\hline 7 & TELESUCESOS & 41 & $\mathrm{M}$ & 29 \\
\hline 8 & 46 UHF ABC & 43 & $\mathrm{M}$ & 46 \\
\hline 9 & CANAL UNO & 45 & $\mathrm{M}$ & 12 \\
\hline
\end{tabular}

El canal virtual es el canal lógico (programable en las tablas) en el que se presenta la señal ISDB-Tb en el receptor de televisión digital.

Las 23 estaciones de TDT, autorizadas para emitir señal, entraron en operación durante el año 2014, y las 9 estaciones autorizadas para operar en la ciudad de Quito se encuentran en un período de simulcast con la señal de televisión analógica, simulcast que según el Plan Maestro de Televisión Digital aprobado por el ex CONATEL, debería terminarse a finales del año 2016. Figura 3b.

${ }^{4}$ Según la Norma Técnica de Televisión Analógica - ARCOTEL 2015, al Grupo G1 corresponden los canales 14, 21, 23, 25, 27, 29, 31, 33 y 35 y al Grupo G4 los canales $38,40,42,44,46,48$ y 50 . 


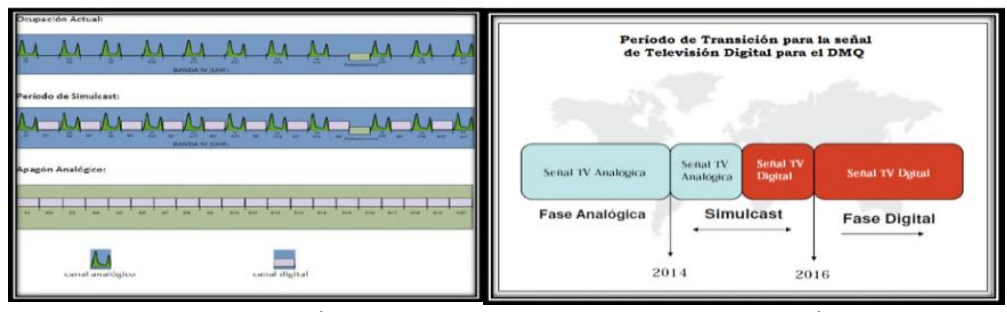

a)

b)

Figura 3 a) Escenario de la Implementación de la TDT; b) Período de Transición para la TDT en el DMQ.

\section{SISTEMA DE ALERTA DE EMERGENCIA CON TELEVISIÓN DIGITAL}

La propuesta resume las normas del Sistema de Emisión de Alerta de Emergencia (EWBS) que se aplican en común a todos los países que adoptaron la norma ISDB-T y comprenden las señales transmitidas por las estaciones emisoras y la interpretación que hacen los receptores compatibles. Ha sido redactada basándose en la norma operativa del EWBS utilizada en Japón con algunas modificaciones para poder cumplir con los requerimientos de los países latinoamericanos y en los documentos de los Foros de ISDB-T aprobados en la reunión de Uruguay en mayo del 2013.

\subsection{Generalidades de la función del EWBS}

Las señales del EWBS transmitidas por los radiodifusores activan los receptores habilitados y muestran la información de emergencia. Consisten en un indicador de activación en la TMCC (Transmission and Multiplexing Configuration Control) y en un descriptor de información de emergencia en la PMT (Program Map Table).

Los radiodifusores comunican la información con; 1) Transmiten el mensaje de texto mediante la función de Superposición (operación obligatoria), 2)Transmiten el programa (operación recomendada)

Del lado del receptor, la señal del EWBS activa los receptores según el código de área y conmuta a la información de emergencia, es decir, interrumpe y muestra los datos de superposición y el programa.

\subsection{Procedimiento de transmisión del EWBS}

\subsubsection{Generalidades de transmisión}

El funcionamiento del EWBS, para su recepción más estable, debería depender de la capa de transmisión más robusta.

Para iniciar el EWBS se debe contar con los siguientes 4 elementos:

1.- El descriptor de información de emergencia que especifica las condiciones del EWBS relacionadas con el indicador de inicio/fin, el ID del servicio y el código de área debe estar presente en el PMT para todos los servicios. 
2.- El radiodifusor debe colocar el indicador de activación para el EWBS en la señal de TMCC en "1".

3.- Un mensaje de texto que se reconoce como información de emergencia por utilizar superposición debe transmitirse en un servicio que transmita información de emergencia.

4.- El programa con información de emergencia, si está disponible, debe transmitirse en un servicio que transmita información de emergencia.

Cuando se detenga el funcionamiento del EWBS, deben transmitirse las señales siguientes:

1.- Se debe colocar en "0" el indicador de activación para EWBS.

2.- Se debe borrar del PMT el descriptor de información de emergencia.

\subsubsection{Señalización en la tabla PMT}

El descriptor de información de emergencia debe utilizarse cuando sean los radiodifusores quienes transmitan el EWBS. Su transmisión es obligatoria y debe hacerse en la PMT. Dado que se transmiten diferentes tablas PMT.

\subsubsection{Modificación del descriptor de información de emergencia}

Mientras está en funcionamiento el EWBS, si es necesario cambiar alguna información, como por ejemplo el código de área, deberá aplicarse el procedimiento completo para detener el EWBS. Después de modificar la información de emergencia el indicador de activación en TMCC debe colocarse en "1" nuevamente. La figura 4 presenta el ejemplo de funcionamiento de transmisión del EWBS de un sistema con cuatro servicios: HD, SD1, SD2 y One-seg.

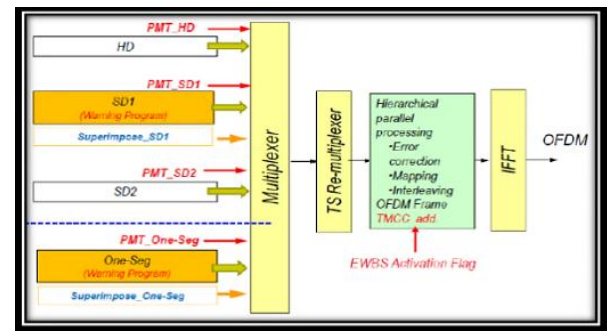

Figura 4 Funcionamiento de EWBS. Fuente: ISDB-T Internacional ${ }^{5}$

\subsection{Procedimiento de recepción del EWBS}

\subsubsection{Generalidades de recepción}

La recepción del EWBS no es una especificación obligatoria para los receptores ISDB-T comunes. Sin embargo, teniendo en cuenta el importante papel del EWBS en la prevención de desastres, se requiere el esfuerzo de que tantos

${ }^{5}$ Fuente ISDB-T Internacional 
receptores ISDB-T como sea posible soporten la función EWBS. Los receptores habilitados para recibir EWBS deben soportar la especificación que se indica a continuación.

Tabla 4 Especificaciones para recepción del EWBS.

\begin{tabular}{|l|c|c|c|c|}
\hline \multirow{2}{*}{ FUNCIÓN } & \multicolumn{2}{c|}{ Receptores Fijos } & \multicolumn{2}{c|}{ Receptores portátiles } \\
\cline { 2 - 5 } & TV & STB & One-seg & EWBS exclusivo \\
\hline Arranque automático & Opcional & Opcional & Recomendado & Obligatorio \\
\hline $\begin{array}{l}\text { Código de área pre } \\
\text { configurado }\end{array}$ & Obligatorio & Obligatorio & Obligatorio & Obligatorio \\
\hline Superposición & Obligatorio & Obligatorio & Obligatorio & Obligatorio \\
\hline Decodificación de programa & Obligatorio & Obligatorio & Obligatorio & - \\
\hline
\end{tabular}

El procedimiento de recepción del EWBS comprende los siguientes pasos:

- El receptor habilitado para recibir EWBS debe monitorear continuamente el indicador de activación en la señal TMCC en modo "stand-by"

- Cuando el indicador de activación en la señal TMCC cambia de "0" a "1", los receptores deben controlar el descriptor de información de emergencia en la PMT del flujo de transporte recibido.

- Cuando el indicador de activación esté en "1" y el código de área de la PMT coincida con el código de área configurado en el receptor, se activarán los receptores. Los receptores deberán entonces mostrar la información de emergencia (Superposición y programa).

- Cuando el indicador de activación está en "0", se trata de una transmisión de prueba y no se requiere ninguna operación en especial.

Los receptores deben monitorear continuamente la PMT mientras el indicador de activación en la TMCC siga en "1". Cuando el "indicador de activación" en la TMCC conmute a "0" y se borre el descriptor de información de emergencia el funcionamiento del EWBS ha llegado a su fin.

\subsubsection{Función de arranque automático}

Se recomienda que los receptores habilitados para recibir EWBS tengan función de arranque automático. Sin embargo, a fin de diseñar estos receptores se debe tener en cuenta lo siguiente:

- El arranque simultáneo de muchos receptores que funcionan con CA podrá dañar o sobrecargar las redes de provisión de energía eléctrica, convirtiendo esta alternativa de alerta en perjudicial antes que en beneficiosa. Es por eso que se recomienda que los dispositivos que consumen mucha energía, como una TV, no tengan la función de arranque automático. En su lugar, se recomienda que estos dispositivos tengan un método de aviso alternativo como un sonido /buzzer/ o una luz intermitente etc., que indique a los usuarios que enciendan la TV.

- En caso de que haya STB no es razonable que la TV conectada arranque automáticamente por la razón antemencionada. Se 
recomienda, por lo tanto, que estos dispositivos tengan un método de aviso alternativo como un sonido o una luz intermitente etc., que indique a los usuarios que enciendan la TV.

- Se recomienda que los receptores portátiles que funcionan con batería portátil tengan función de arranque automático.

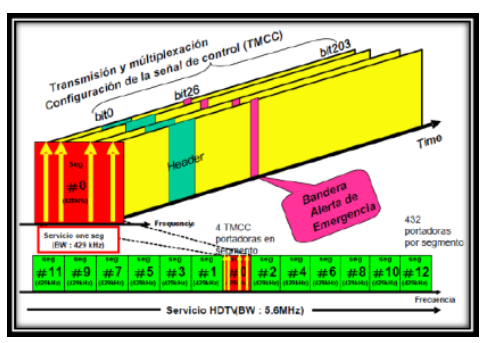

a)

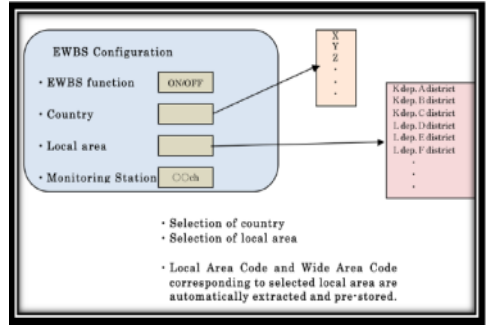

b)

Figura 5 a) Ubicación de la bandera de alerta de emergencia en la trama ISDB-T. Fuente: ISDB-T Internacional; b) Configuración de receptores de TDT para aceptar señal EWBS. Fuente: ISDB-T Internacional

\subsection{Pre configuración de datos}

\subsubsection{Código de área}

El código de área estará pre configurado según la configuración propia del usuario. Por ejemplo, los usuarios podrán configurar su administración zonal al momento de la instalación y los receptores lo tomarán y adjudicarán al código de área apropiado asignado para el funcionamiento del EWBS en cada país.

Al momento de la instalación, debe pre configurarse al mismo tiempo la información del país donde se encuentran los receptores.

De la figura 5b, que es un ejemplo de lo implementado en Japón para el caso de equipos que cuentan con la alternativa de EWBS, el usuario que adquiera uno de estos equipos debería programarlo dependiendo del área donde lo va a utilizar de manera que ese equipo quede apto para recibir señales EWBS. La información que se debería programar sería:

1) Activar la función EWBS. 2) Escoger el país, aunque para evitar conflictos en el caso que el equipo receptor sea usado en otro país que tenga adoptada la norma ISDB-Tb, lo aconsejable sería que este campo sea tomado automáticamente de la información emitida por el canal designado para levantar la alerta. 3) Ingresar el código de área local, que en el caso del DMQ podría ser la Administración Zonal a la que corresponda la vivienda. 4) Finalmente se debe configurar el canal de monitoreo de alerta, mismo que será comunicado obligatoriamente al momento de adquirir el equipo.

\subsubsection{Estación de monitoreo en modo stand-by}

Para la función de arranque automático del EWBS, los receptores deben monitorear continuamente el indicador de activación en la señal TMCC en modo stand-by. Teniendo en cuenta que los receptores solo pueden monitorear una 
estación de radiodifusión, debe programarse una estación de monitoreo específica, misma que será definida por los Organismos pertinentes con la debida oportunidad.

Como principio, se recomienda que los usuarios puedan configurar por sí mismos la estación de monitoreo. (La estación de monitoreo por defecto debe ser la misma que cuando se apagó el receptor la última vez) Para que esto sea posible las organizaciones responsables deben informar a los usuarios por adelantado cuáles estaciones de radiodifusión operan el EWBS.

Al buscar señales RF durante la instalación inicial, los receptores deben verificar el "código de país" en "el descriptor de compensación de hora local" de cada señal RF. La transmisión del "código de país" en el "descriptor de compensación de hora local" es obligatoria para todas las estaciones de radiodifusión ISDB-T para asegurar el funcionamiento correcto del EWBS.

\subsubsection{Indicador de activación}

La información de la TMCC ayudará al receptor a desmodular y decodificar información variada, incluyendo: identificación del sistema, indicación de transmisión-conmutación de parámetro, indicador de activación del EWBS, información de la configuración actual y futura. Cuando se conmuta solamente el indicador de activación del EWBS, el contenido del indicador de la conmutación de parámetro de transmisión no retrocederá.

Al indicador de activación del EWBS se le asigna el bit 26 de la TMCC, B26. El contenido del indicador de activación será 1 cuando el arranque del receptor esté controlado y 0 cuando no esté controlado.

Tabla 5 Significado del bit de activación de EWBS en la TMCC.

\begin{tabular}{|c|c|}
\hline $\mathbf{B}_{\mathbf{2 6}}$ & Significado \\
\hline 0 & Sin control de arranque \\
\hline 1 & Con control de arranque \\
\hline
\end{tabular}

Los radiodifusores deben mantener el indicador de activación de la señal TMCC configurado en "1" mientras se transmite información de emergencia independientemente de la capa de transmisión en que se transporta el servicio que envía el EWBS.

\subsubsection{Descriptor de información de emergencia} siguiente:

La semántica para el descriptor de información de emergencia será la

- Servicio_id: campo de 16 bits que indicarán el número de evento de radiodifusión. Será el mismo que el número del programa.

- Indicador de inicio-fin: campo de 1 bit que corresponderá a la señal de inicio y de fin del funcionamiento del EWBS. Cuando el bit esté en "1" significa que el EWBS ya fue iniciado o está en funcionamiento. Cuando esté en " 0 " significa que finalizó;

- Nivel de señal: campo de 1 bit que corresponde a la señal de alarma de emergencia especificada por las agencias responsables. Cuando 
esté en "0", significa que la señal de alarma de emergencia es el primer tipo de señal de inicio. Cuando esté en "1" la señal de alarma será el segundo tipo de señal de inicio. No debe usarse esta información para controlar a los receptores habilitados para recibir el EWBS.

- Longitud de código de área: campo de 8 bits que indica el tamaño en bytes del código de área.

- Código de área: campo de 12 bits que corresponda al código de área especificado por las agencias responsables.

La información de código de área es también parte del descriptor del sistema de entrega terrestre que indica las condiciones físicas de la transmisión terrestre.

\subsubsection{Superposición}

La información de alerta de emergencia tiene prioridad sobre otros contenidos para la transmisión de superposición.

Los servicios de superposición se transmiten a través de una PES (Packetized Elementary Stream) no sincronizada, en otras palabras, no se envía la información PTS. Las siguientes restricciones aplican a la superposición:

- Número máximo de idiomas por 1 ES: 1 idioma;

- Tamaño máximo de PES: 32 KB;

- Intervalo mínimo para envío de paquetes PES: 100 ms;

- Velocidad máxima ES: $256 \mathrm{Kbit} / \mathrm{s}$;

- Buffer receptor: mayor o igual a $64 \mathrm{~KB}$.

La superposición se transporta por una PES independiente identificada en la PMT con los valores de acuerdo a la tabla 7.

Tabla 6. Señalización para la superposición de textos con señal EWBS.

\begin{tabular}{|c|c|c|}
\hline Descripción & Tipo de flujo & $\begin{array}{c}\text { Etiqueta del } \\
\text { Componente }\end{array}$ \\
\hline Superposición para full-seg & \multirow{2}{*}{$0 \times 06$} & $0 \times 38$ \\
\cline { 1 - 1 } Superposición para one-seg & & $0 \times 88$ \\
\hline
\end{tabular}

a)

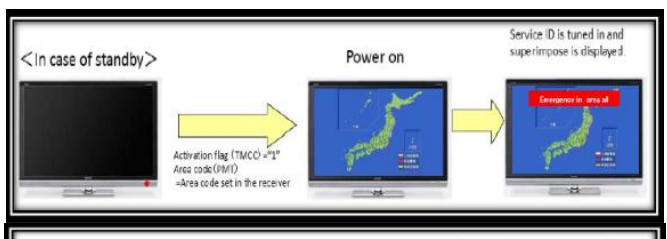

b)

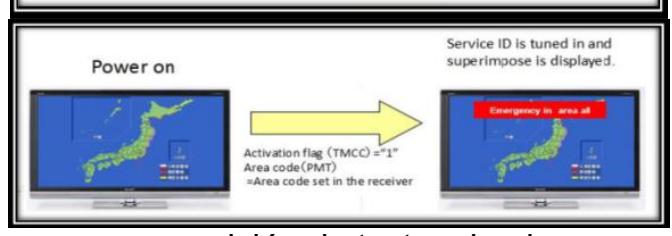

Figura 6 a) Encendido con superposición de textos desde un receptor en "stand by". Fuente: SHARP CORPORATION; b) Encendido con superposición de textos desde un receptor en "on". Fuente: SHARP CORPORATION 
La Figura 6 a) y b) muestran la activación de un receptor de televisión.

\section{SISTEMA DE ALERTA DE EMERGENCIA PARA EL DISTRITO METROPOLITANO DE QUITO}

Una vez analizadas todas las variables, se plantea la alternativa para la instalación del Sistema de Alerta de Emergencia para el DMQ, para ello, se establece primero la propuesta de zonificación a través de códigos a ser transmitidos, de manera que se informe solamente a los sectores que pueden ser afectados, después se realiza un análisis de los canales de televisión que podrían difundir esta alerta de emergencia, dependiendo de su área de cobertura y su infraestructura física y tecnológica, finalmente se establecerá la propuesta de red de emergencia sobre la base de experiencias internacionales.

\subsection{Zonificación y códigos de área}

Para la propuesta de zonificación y códigos de área se parte de los documentos e información presentada por varios países que integran el Foro ISDBT, en la reunión llevada a cabo en Uruguay en el 2013, este Foro agrupa a todos los países que han adoptado al estándar brasileño - japonés como estándar de televisión digital ${ }^{6}$. Dentro de estos Foros Internacionales, existen los grupos de trabajo denominados de "Armonización" a los que se les encarga los diferentes temas a ser tratados, es así que en el Foro Internacional llevado a cabo en Montevideo - Uruguay en mayo del $2013^{7}$, se hicieron algunas propuestas respecto a la posibilidad de incorporar alertas de emergencia dentro de la señal de televisión digital, en estos ejercicios se plantearon ideas interesantes que se recogen para el planteamiento ecuatoriano. Una de esas ideas, fue la de definir los códigos para cada país partiendo de las divisiones territoriales que internamente se administren en los mismos. Para el presente caso se parte de la división territorial que ha realizado el INEC después del censo del 2010, en la que se ha determinado que el Ecuador está dividido territorialmente en 24 provincias, divididas a su vez en 224 cantones, de ahí que para la propuesta de codificación se considerará la determinación de un código local, luego los códigos provinciales y luego el nacional. Para el caso de la última capa que se refiere a los cantones, los códigos se compondrán de un código decimal "provincia" seguido de otro código decimal "cantón". En las siguientes tablas se desarrolla la propuesta de codificación.

${ }^{6}$ A la fecha los países que han adoptado el estándar ISDB-T en el mundo son; Japón, Brasil, Perú, Argentina, Chile, Venezuela, Ecuador, Costa Rica, Paraguay, Filipinas, Bolivia, Belice, Islas Maldivas, Guatemala, Uruguay, Nicaragua y Botsuana.

7 En el Foro ISDB-T de Uruguay del 2013, estuvieron presentes delegados de Argentina, Bolivia, Botsuana, Brasil, Costa Rica, Chile, Ecuador, Filipinas, Japón, Paraguay, Perú, Uruguay y Venezuela. 
Tabla 7 Propuesta de numeración de los cantones de Pichincha.

\begin{tabular}{|l|c|c|c|c|c|}
\hline \multicolumn{1}{|c|}{ Cantón } & $\begin{array}{c}\text { Número } \\
\text { (1 al 224 - INEC) }\end{array}$ & $\begin{array}{c}\text { Número } \\
\text { (Ordinal) }\end{array}$ & Provincia & $\begin{array}{c}\text { Número } \\
\text { (1 AL 24 - } \\
\text { INEC) }\end{array}$ & $\begin{array}{c}\text { Número } \\
\text { para código }\end{array}$ \\
\hline Cayambe & 30 & 01 & Pichincha & 17 & 1701 \\
\hline Mejía & 108 & 02 & Pichincha & 17 & 1702 \\
\hline Pedro Moncayo & 145 & 03 & Pichincha & 17 & 1703 \\
\hline $\begin{array}{l}\text { Pedro Vicente } \\
\text { Maldonado }\end{array}$ & 146 & 04 & Pichincha & 17 & 1704 \\
\hline Puerto Quito & 158 & 05 & Pichincha & 17 & 1705 \\
\hline Quito & 168 & 06 & Pichincha & 17 & 1706 \\
\hline Rumiñahui & 172 & 07 & Pichincha & 17 & 1707 \\
\hline $\begin{array}{l}\text { San Miguel de } \\
\text { los Bancos }\end{array}$ & 183 & 08 & Pichincha & 17 & 1708 \\
\hline
\end{tabular}

Tabla 8 Propuesta de Codificación de Capa 1 - Área Local 1 - Cantonal

\begin{tabular}{|l|c|c|}
\hline \multicolumn{1}{|c|}{ Cantón } & $\begin{array}{c}\text { Código de Área } \\
\text { (Decimal) }\end{array}$ & $\begin{array}{c}\text { Código de Área } \\
\text { (Binario) }\end{array}$ \\
\hline Cayambe & 1701 & 011010100101 \\
\hline Mejía & 1702 & 011010100110 \\
\hline Pedro Moncayo & 1703 & 011010100111 \\
\hline Pedro Vicente Maldonado & 1704 & 11010101000 \\
\hline Puerto Quito & 1705 & 011010101001 \\
\hline Quito & 1706 & 011010101010 \\
\hline Rumiñahui & 1707 & 011010101011 \\
\hline San Miguel de los Bancos & 1708 & 011010100100 \\
\hline
\end{tabular}

Tabla 9. Propuesta de Codificación de Capa 2 - Área Amplia 1 - Provincial

\begin{tabular}{|c|c|c|}
\hline Provincia & $\begin{array}{c}\text { Código de Área } \\
\text { (Decimal) }\end{array}$ & $\begin{array}{c}\text { Código de Área } \\
\text { (Binario) }\end{array}$ \\
\hline Pichincha & 1700 & 011010100100 \\
\hline
\end{tabular}

Tabla 10. Propuesta de Codificación de Capa 3 - Área Amplia 2 - Nacional.

\begin{tabular}{|c|c|c|}
\hline País & $\begin{array}{c}\text { Código de Área } \\
\text { (Decimal) }\end{array}$ & $\begin{array}{c}\text { Código de Área } \\
\text { (Binario) }\end{array}$ \\
\hline Ecuador & 1000 & 001111101000 \\
\hline
\end{tabular}

Esta propuesta de codificación permite diferenciar entre las áreas de la provincia de Pichincha, y del DMQ, que podrían verse afectadas en el caso de la ocurrencia de una amenaza natural o antrópica, de manera que en el caso de emitirse una señal de alerta a través de la señal de televisión digital, solo la población de los cantones que los Organismos pertinentes establezcan como posibles zonas de afectación serían alertados a través de esta tecnología. De la misma manera en la propuesta se establece un código provincial que dispararía las alertas en toda la provincia y uno nacional en el caso de ser necesario. 


\subsection{Canales de televisión que pueden levantar el sistema de alerta de emergencia}

Revisando el listado de todas las estaciones de televisión autorizadas en el país, se considerarán aquellas que tienen dentro de su área de cobertura al Distrito Metropolitano de Quito ${ }^{8}$, y luego el número de repetidoras de cada una de ellas, de manera de estimar aquellas que cubrirían el mayor número de cantones, teniendo en cuenta además que en la mayoría de casos, cada estación repetidora no cubre únicamente un cantón sino varios de ellos, e inclusive en algunos casos llegan a cubrir dos provincias desde una misma repetidora; esta información se compara con aquellas estaciones que tienen al aire una señal de televisión digital en la ciudad de Quito para elaborar la tabla 15 que indica las estaciones de televisión de la ciudad de Quito, que tienen al aire una señal analógica y una señal digital, ordenadas por el número de repetidoras que tienen autorizadas:

Tabla 11. Canales de TV que operan en el DMQ, que cuentan con señal de TDT, ordenados por número de repetidoras en el territorio nacional.

\begin{tabular}{|l|c|c|c|}
\hline \multicolumn{1}{|c|}{ ESTACIÓN } & CANAL & $\begin{array}{c}\text { NUMERO DE } \\
\text { REPETIDORAS }\end{array}$ & $\begin{array}{c}\text { SEÑAL DE TDT } \\
\text { (EN LA MATRIZ) }\end{array}$ \\
\hline ECUADOR TV & 7 & 98 & $\mathrm{SI}$ \\
\hline TELEVISIÓN DEL PACIFICO & 2 & 50 & $\mathrm{SI}$ \\
\hline TELESISTEMA & 5 & 32 & $\mathrm{SI}$ \\
\hline TELEAMAZONAS & 4 & 30 & $\mathrm{SI}$ \\
\hline CANAL UNO & 12 & 17 & $\mathrm{SI}$ \\
\hline TELEVISIORA NACIONAL & 8 & 9 & $\mathrm{SI}$ \\
\hline TELEVISIÓN SATELITAL & 25 & 3 & $\mathrm{SI}$ \\
\hline 46 UHF ABC (RTU) & 46 & 1 & $\mathrm{SI}$ \\
\hline TELESUCESOS & 29 & 0 & $\mathrm{SI}$ \\
\hline
\end{tabular}

Como todas las estaciones listadas anteriormente han sido instaladas con transmisores que cumplen el estándar ISDB-Tb, cualquiera de ellas podría servir para levantar una señal de alerta de emergencia; considerando la cobertura que tiene ECUADOR TV con casi 100 estaciones operando al momento en el país, que los equipos de TDT del canal fueron instalados por técnicos japoneses, que el personal técnico del canal fue capacitado tanto en Quito como en Japón sobre el funcionamiento de los equipos instalados (sobre la base de un convenio de cooperación que se suscribió al decidir por el estándar ISDB-Tb), y sobre todo porque la naturaleza del canal es de servicio al público, además de ser el canal oficial del Gobierno Ecuatoriano, se considera que ECUADOR TV es la mejor alternativa para levantar el Sistema de Alerta de Emergencia. A continuación se presenta la figura 7 con el área de cobertura que tendría la señal de TDT de ECUADOR - TV, desde el cerro Pichincha, obtenida con ICS Telecom.

${ }^{8}$ Según el listado publicado por ARCOTEL el área autorizada comprende: QUITODISTRITO METROPOLITANO, SANGOLQUI, MACHACHI, CAYAMBE, TABACUNDO. 


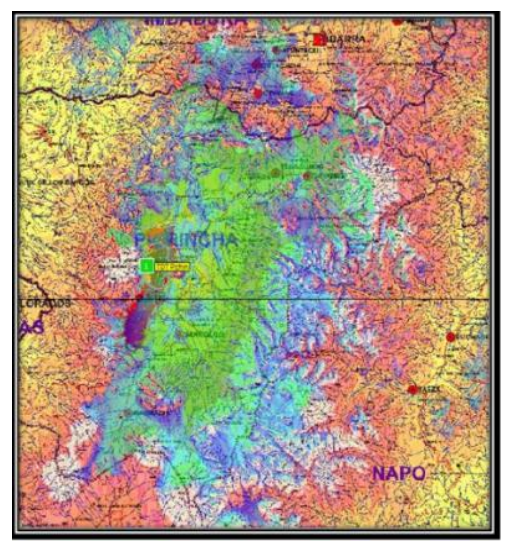

Figura 7 Área de cobertura de un canal de TDT para el DMQ. Fuente:

\section{SUPERTEL ${ }^{9}$}

\subsection{Instituciones involucradas en monitoreo, gestión y difusión de amenazas en el DMQ}

El análisis de los Organismos e Instituciones relacionados con la ocurrencia de las amenazas naturales y antrópicas que pueden afectar a al DMQ, se coloca dentro de un diagrama de operación que resume su accionar.

\subsection{Vigilancia y monitoreo}

Para el caso del DMQ esta responsabilidad la tendrían a cargo las siguientes instituciones:

El Instituto Geofísico de la EPN, es el encargado de monitorear y vigilar permanentemente las amenazas sísmicas y volcánicas en el Ecuador, por tanto quien informaría a los Organismos competentes.

La Secretaría de Seguridad y Gobernabilidad del DMQ es la encargada de vigilar la ocurrencia de eventos naturales y antrópicos en la jurisdicción del DMQ, excepto amenazas sísmicas y volcánicas, por lo tanto será la encargada de informar a los Organismos competentes.

\subsection{Gestión de las amenazas}

A cargo de este proceso se encuentra la Secretaría Nacional de Gestión de Riesgos - SNGR, que es la que establece las políticas, regulaciones y lineamientos estratégicos de gestión de riesgos que incluye la prevención, mitigación, preparación, respuesta, rehabilitación, reconstrucción, recuperación y transferencia del riesgo, así como de la generación de alertas tempranas.

\subsection{Difusión de amenazas naturales y antrópicas}

Este proceso lo lidera el Ministerio Coordinador de Seguridad, encargado en el Ecuador de emitir las políticas de seguridad que deban implementarse en el caso

${ }^{9}$ Simulación de cobertura realizada con el programa ICS Telecom - 2014. 
de emergencias que pudieran afectar a los ciudadanos, así como de coordinar la acción de las diferentes instituciones relacionadas con este tema en el país.

\subsection{Operación de la red de alerta de emergencia a través de la TDT para el DMQ}

Del análisis realizado a lo largo del presente estudio, se plantean los actores en la Red de Alerta de Emergencia, con funciones y responsabilidades a definirse conforme a su ámbito de acción.

- Ministerio Coordinador de la Seguridad

- Secretaría Nacional de Gestión de Riesgos

- Instituto Geofísico de la EPN

- Secretaría de Seguridad del DMQ

- Canal designado (Ecuador TV)

- Sociedad Civil (Población del DMQ

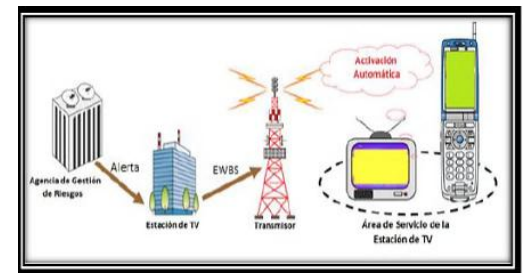

Figura 8 Red de alerta de emergencia a través de TDT en el DMQ.

Fuente: ISDB-T Internacional

\section{CONCLUSIONES Y RECOMENDACIONES}

\subsection{Conclusiones}

El uso de las subportadoras de televisión digital para la implementación de una red de alerta de emergencia para el Distrito Metropolitano de Quito y su zona de influencia en la que se incluyen los valles de Tumbaco, Cumbayá y Los Chillos es una alternativa tecnológica que debe ser tomada en cuenta por los Organismos responsables de la seguridad ciudadana, considerando sobre todo que al ser la televisión digital una tecnología que está empezando a implementar, su vigencia tecnológica está garantizada a largo plazo.

Con la información obtenida de la Secretaría de Seguridad del DMQ se pudieron identificar las zonas y alrededores que podrían ser afectados por la ocurrencia de amenazas naturales y antrópicas, mismas que, según la predicción de la señal de televisión digital realizada, estarían dentro de la cobertura de la estación de televisión que emitiría la señal de alerta de emergencia, con lo cual se puede afirmar, todo el territorio del DMQ sería cubierto en forma simultánea.

Considerando las diferentes alternativas tecnológicas que permiten implementar redes de alerta de emergencia en el caso de ocurrir una amenaza natural o antrópica, se puede afirmar que el uso de las subportadoras e infraestructura de TDT, tiene ventajas técnicas y operativas, al ser un sistema complementario con otros sistemas disponibles, además que es la única alternativa 
que combina simultáneamente una gran cobertura con una segmentación de áreas de notificación, además que ya se dispone de infraestructura instalada y que la televisión es el medio de comunicación preferido por los habitantes, por lo que se concluye que el empleo de TDT es la mejor alternativa disponible al momento para la implementación de una red de alerta de emergencia para el Distrito Metropolitano de Quito.

Al estudiar los canales radioeléctricos de la TDT y sus diferentes subportadoras se pudo determinar que en cualquiera de los 13 segmentos, se pueden transmitir datos adicionales sin que se perjudique la señal principal, gracias a la multiplexación de datos que se da en la etapa de transmisión, sin embargo, por estandarización, se sugiere el uso del segmento 0 ya que alcanza a una mayor gama de receptores, incluyendo televisores digitales, set top box y dispositivos móviles.

Al analizar la forma en que los datos pueden ser insertados en una subportadora de TDT para implementar una red de alerta de emergencia para el Distrito Metropolitano de Quito y sus zonas de influencia, se encontró que el Sistema de Emisión de Alerta de Emergencia (EWBS por sus siglas en inglés) se puede aplicar en común para todos los países que adoptaron la norma ISDB-T, por lo que además de compartir experiencias y avances sobre esta temática, se podrían aplicar también economías de escala en la fabricación de dispositivos (hardware y software) que permitan la generación y transmisión de mensajes de alerta de emergencia por parte de las estaciones emisoras y la recepción y decodificación de dichos mensajes, segmentando la activación y el encendido racional de los dispositivos.

\subsection{Recomendaciones}

Es importante que previo a tomar una decisión sobre la implementación de un sistema de alerta de emergencia que pueda ser utilizado no solo en el DMQ sino en cualquier parte del país, se establezca por parte del Ministerio Coordinador de Seguridad una comisión donde participen delegados de los Organismos identificados en el presente estudio, que evalúen las diferentes propuestas planteadas y la factibilidad de implementarlas progresivamente en diferentes sectores del país, solas o como complemento a otras alternativas como son las sirenas instaladas actualmente en el sur del DMQ y en el cantón Rumiñahui por la posible erupción del volcán Cotopaxi, considerando sobre todo las ventajas que tendría la alternativa de utilizar la TDT para estos sistemas de alerta como son: una mayor cobertura, una difusión simultánea masiva, la segmentación de zonas afectadas y el uso de infraestructura ya instalada, características que no tiene ningún otro sistema.

De producirse una amenaza natural o antrópica es fundamental llegar a las poblaciones que puedan ser afectadas de manera oportuna por lo que para la difusión de un mensaje de alerta de emergencia se deberían utilizar todos los medios disponibles de manera que entre ellos se complementen para cubrir a la mayor cantidad de población y en el menor tiempo posible, por ello se sugiere que además de las alertas que puedan emitirse a través de la señal de TDT, de así decidirse y previo las autorizaciones y coordinaciones necesarias con los Organismos encargados, se emitan también mensajes a través de las señales analógicas de 
radio y televisión, mensajes cortos (SMS) a través de la telefonía móvil, sirenas e inclusive mensajes usando otras alternativas técnicas de broadcast como son los datos RDS de las estaciones de radiodifusión sonora designadas.

\section{REFERENCIAS}

1. D’Ercole - Metzger, R. - P.; (2004). La Vulnerabilidad del Distrito Metropolitano de Quito. Municipio del Distrito Metropolitano de Quito, Dirección Metropolitana de Territorio y Vivienda.

2. Secretaria de Seguridad y Gobernabilidad, Municipio del Distrito Metropolitano de Quito; (2015). Atlas de Amenazas Naturales y Exposición de Infraestructura del Distrito Metropolitano de Quito. Segunda Edición.

3. Jacks - Davidson - Wai, E. - J. - H.; (2010). Directrices Sobre Sistemas de Alerta Temprana y Aplicación de Predicción Inmediata y Operaciones de Aviso. Organización Meteorológica Mundial (OMM).

4. Basher, R.; (2006). Global Early Warning System for Natural Hazards: Systematics and People - Centred. Philosophical Transaction of the Royal Society.

5. Hall, P.; La Red (Red de Estudios Sociales en Prevención de Desastres en América Latina). (2006). Early Warning Systems: Reframing the Discussion. Recuperado el 15 de febrero de2016, de http://www.desenredadando.org/public/articulos/2006/sis_alert Temp/EWS_Reframing_the discussion.pdf.

6. Peltre, P.; (1989). Riesgos Naturales en Quito, Lahares, Aluviones y Derrumbes del Pichincha y del Cotopaxi. Corporación Editora Nacional.

7. DiBEG - Digital Broadcasting Experts Group; (2009). Contenidos Técnicos y Estructura del Sistema ISDB-T. Recuperado el 18 de febrero de 2016, de http://www.dibeg.org/techp/feature/ANNEX-AA_spanish.pdf.

8. Ivo - Queiroz, R. - R.; (2008). Sistema de Radiodifusión Digital Terrestre. Kathrein Mobilcom Brasil. Dinámica Gráfica y Editora. Segunda Edición.

9. Fisher, W.; (2008). Tecnologías para la Radiodifusión Digital de Audio y Video. Springer - Verlag Berlín. Segunda Edición.

10. Sotelo - Durán - Joskowics. Universidad de Montevideo; (2011). Sistema de Transmisión ISDB-T. Recuperado el 4 de marzo de 2016, de http://www.um.edu.uy/_upload/_descarga/web_descarga_240_Sistemadet ransmisinl SDB-T.-Sotelo_Durn_Joskowicz.pdf

11. Pisciota, N.; Universidad Blas Pascal. (2010). Sistema ISDB-Tb (Primera Parte). Recuperado el 8 de marzo de 2016, de http://www.ubp.edu.ar/wp- content/uploads/2013/12/392010MI-SistemaISDB-Tb-Primera-Parte.pdf

12. Embajada del Japón en Argentina - DiBEG; (2011). Transmisión de Televisión Digital Terrestre - ISDB-T Integrated Services Digital Broadcasting Terrestrial. Recuperado el 12 de marzo 2016, de japan.go.jp/Notas/090901TelevisionDigital.pdf

13. Instituto Nacional de Telecomunicações - INATEL; (2012). Curso Introdução ao Sistema de Televisão Digital. Módulos 2 al 5.

14. Instituto Nacional de Telecomunicações - INATEL; (2013). Curso Televisão Digital Avançãdo, Da Teoria á Prática. Módulos 1 al 3. 
15. Sakaguchi, Y.; NHK - Nippon Hoso Kyokai. (2014). Role of ISDB-T in Disaster Reduction. JICA Training Course. Engineering Administration Department, NHK, Japan.

16. NEC Corporation. (2014). Emergency Warning Broadcasting System. JICA Training Course. Japan.

17. SHARP Corporation. (2014). Emergency Warning Receiver based on OneSegmentation Broadcast System. Engineering Department. JICA Training Course. Japan.

18. Sugihara, Y.; NHK - Nippon Hoso Kyokai. (2014). Broadcasting Systems for Emergency. JICA Training Course. News productions \& Network Department, NHK, Japan.

19. Superintendencia de Telecomunicaciones - SUPERTEL. (2009). Encuesta de uso, hábitos y preferencias de la televisión en el Ecuador.

20. Consejo Nacional de Telecomunicaciones - CONATEL. (2012). Plan Maestro de Televisión Digital Terrestre.

21. Agencia de Regulación y Control de las Telecomunicaciones - ARCOTEL. (2015). Norma Técnica para el Servicio de Radiodifusión de Televisión Abierta Analógica. 